\title{
The Gerber-Shiu Expected Penalty Function for the Risk Model with Dependence and a Constant Dividend Barrier
}

\author{
Donghai Liu, ${ }^{1}$ Zaiming Liu, ${ }^{2}$ and Dan Peng ${ }^{1}$ \\ ${ }^{1}$ Department of Mathematics, Hunan University of Science and Technology, Xiangtan, Hunan 411201, China \\ ${ }^{2}$ Department of Mathematics, Central South University, Changsha, Hunan 410075, China \\ Correspondence should be addressed to Donghai Liu; dhliu@hnust.edu.cn
}

Received 12 June 2014; Revised 11 July 2014; Accepted 11 July 2014; Published 23 July 2014

Academic Editor: Dumitru Baleanu

Copyright (c) 2014 Donghai Liu et al. This is an open access article distributed under the Creative Commons Attribution License, which permits unrestricted use, distribution, and reproduction in any medium, provided the original work is properly cited.

\begin{abstract}
We consider a compound Poisson risk model with dependence and a constant dividend barrier. A dependence structure between the claim amount and the interclaim time is introduced through a Farlie-Gumbel-Morgenstern copula. An integrodifferential equation for the Gerber-Shiu discounted penalty function is derived. We also solve the integrodifferential equation and show that the solution is a linear combination of the Gerber-Shiu function with no barrier and the solution of an associated homogeneous integrodifferential equation.
\end{abstract}

\section{Introduction}

In the classical compound Poisson risk model, the surplus process has the form

$$
U(t)=u+c t-\sum_{i=1}^{N(t)} X_{i}, \quad t \geq 0,
$$

where $u \geq 0$ is the initial surplus, $c \geq 0$ is the premium income rate, and $\left\{X_{i}\right\}_{i=1}^{\infty}$ are i.i.d. random variables representing the individual claim amounts with probability density function (p.d.f.) $f_{X}$, cumulative distribution function (c.d.f.) $F_{X}$, and Laplace transform (LT) $f_{X}^{*}$. The counting process $\{N(t) ; t \geq 0\}$ denotes the number of claims up to time $t$ and is defined as $N(t)=\max \left\{k: W_{1}+W_{2}+\right.$ $\left.\cdots+W_{k} \leq t\right\}$, where the interclaim times $\left\{W_{i}, i=1,2, \ldots\right\}$ form a sequence of independent and strictly positive realvalued random variables (r.v.s.). The r.v. $\left\{W_{i}, i=1,2, \ldots\right\}$ have common density function $f_{W}(t)=\lambda e^{-\lambda t}, t>0$, cumulative distribution function $F_{W}$, and Laplace transform $f_{W}^{*} \cdot\{N(t), t \geq 0\}$ is Poisson process with parameter $\lambda>0$.

Ruin probability and related problems in the classical risk model have been studied extensively. Gerber and Shiu [1] introduced a discounted penalty function with respect to the time of ruin, the surplus before ruin, and the deficit at ruin.
Many quantities can be analyzed through this function in a unified manner.

In ruin theory, the classical compound Poisson risk model is based on the assumption of independence between the claim amount random variable $X_{i}$ and the interclaim time $W_{j}$. However, there exist many real-world situations for which such an assumption is inappropriate. For instance, in modeling natural catastrophic events, we can expect that, on the occurrence of a catastrophe, the total claim amount and the time elapsed since the previous catastrophes are dependent. See, for example, Boudreault [2] and Nikoloulopoulos and Karlis [3] for an application of this type of dependence structure in an earthquake context. And as discussed in Albrecher and Teugels [4], they allow the interclaim time and its subsequent claim size to be dependent according to an arbitrary copula structure, by employing the underlying random walk structure of the risk model; they derive exponential estimates for finite- and infinite-time ruin probabilities in the case of light-tailed claim sizes. In Boudreault et al. [5], a risk model with time-dependent claim sizes (i.e., the distribution of the next claim size depends on the last interarrival time) is analyzed and a defective renewal equation for the GerberShiu discounted penalty function is derived and solved. Marceau [6] has considered the discrete-time renewal risk model with dependence between the claim amount random 
variable and the interclaim time random variable. Recursive formulas are derived for the probability mass function and the moments of the total claim amount over a fixed period of time. Cossette et al. [7] use the Farlie-Gumbel-Morgenstern (FGM) copula to define the dependence structure between the claim size and the interclaim time; they derive the integrodifferential equation and the Laplace transform (LT) of the Gerber-Shiu discounted penalty function. An explicit expression for the LT of the discounted value of a general function of the deficit at ruin is obtained for claim amounts having an exponential distribution. Zhang and Yang [8] construct the bivariate cumulative distribution function of the claim size and interclaim time by Farlie-GumbelMorgenstern copula in a compound Poisson risk model perturbed by a Brownian motion. The integrodifferential equations and the Laplace transforms for the Gerber-Shiu functions are obtained. They also show that the Gerber-Shiu functions satisfy some defective renewal equations.

The FGM copula is given by

$$
\begin{array}{r}
C_{\theta}^{\mathrm{FGM}}\left(u_{1}, u_{2}\right)=u_{1} u_{2}+\theta\left(1-u_{1}\right)\left(1-u_{2}\right), \\
0 \leq u_{1}, \quad u_{2} \leq 1,
\end{array}
$$

where $-1 \leq \theta \leq 1$. Note that FGM copula allows both negative and positive dependence, and it also includes the independence copula $(\theta=0)$.

In this paper, we assume that $\left\{\left(X_{i}, W_{j}\right), i \in N^{+}, j \in\right.$ $N^{+}$\} form a sequence of i.i.d. random vectors distributed as the canonical r.v. $(X, W)$. The joint p.d.f. of $(X, W)$ is denoted by $f_{X, W}(x, t)$ with $t \in R^{+}$and $x \in R^{+}$. The joint distribution of $(X, W)$ is defined with a FGM copula; we consider the same dependence risk model with the presence of a constant dividend barrier. We recall that the dividend strategies for insurance risk models were first proposed by De Finetti [9]. Barrier strategies for the compound Poisson risk model have been studied in a number of papers and books, including Landriault [10], Albrecher et al. [11], Yuen et al. [12], Dickson and Waters [13], Lin et al. [14], and Segerdahl [15]. Then, various dividend strategies (threshold dividend strategy, multilayer dividend strategy, etc.) have been studied for different risk models; see, for example, Lin et al. (2006), Chi and Lin [16], D. Liu and Z. Liu [17], Bratiichuk [18], Chadjiconstantinidis and Papaioannou [19], and Wang [20]. As we know, this is the first time to consider the classic risk model with dependence structure based on FGM copula and a constant dividend barrier.

The present paper is organized as follows. In Section 2, the risk model with dependence in the presence of a constant dividend barrier is introduced. And we briefly present some properties of the FGM copula. In Section 3, we derive an integrodifferential equation for the Gerber-Shiu discounted penalty function. Finally, in Section 4, we use a renewal equation to derive an analytical expressions for $m_{b, \delta}(u)$.

\section{Dependence Structure and Risk Model}

A bivariate copula $C$ is a joint distribution function on $[0,1] \times[0,1]$ with uniform marginal distributions. Assume a bivariate random vector $(U, V)$ with above uniform marginal, which has a dependence structure defined by a copula $F_{U, V}=C(u, v)$ with $(u, v) \in[0,1] \times[0,1]$. Important copulas are the independence copula with $C^{\perp}(u, v)=u v$ and the comonotonic copula with $C^{+}(u, v)=\min (u v)$; the countermonotonic copula with $C^{-}(u, v)=\max (u+v-$ $1 ; 0)$. It is important to mention that all copulas satisfy the inequalities $C^{-}(u, v) \leq C(u, v) \leq C^{+}(u, v)$, for $(u, v) \in[0,1] \times$ $[0,1]$.

The joint p.d.f. associated to a copula $C$ is defined by

$$
c\left(u_{1}, u_{2}\right)=\frac{\partial^{2}}{\partial u_{1} \partial u_{2}} C\left(u_{1}, u_{2}\right) .
$$

Let the bivariate distribution function $F_{X, W}$ of $(X, W)$ with marginals $F_{X}$ and $F_{W}$ be defined as $F_{X, W}(x, t)=$ $C\left(F_{X}(x), F_{W}(t)\right)$, for $(x, t) \in R^{+} \times R^{+}$. The joint p.d.f. of $(X, W)$ is given by

$$
f_{X, W}(x, t)=c\left(F_{X}(x), F_{W}(t)\right) f_{X}(x) f_{W}(t),
$$

for $(x, t) \in R^{+} \times R^{+}$(for a survey on copulas we refer the reader to Nelsen [21]).

The FGM copula is given by

$$
\begin{array}{r}
C_{\theta}^{\mathrm{FGM}}\left(u_{1}, u_{2}\right)=u_{1} u_{2}+\theta\left(1-u_{1}\right)\left(1-u_{2}\right), \\
(-1 \leq \theta \leq 1),
\end{array}
$$

where $C_{0}^{\mathrm{FGM}}=C^{\perp}$. So we have

$$
\begin{gathered}
F_{X, W}(x, t)=F_{X}(x) F_{W}(t)+\theta F_{X}(x) F_{W}(t) \\
\times\left(1-F_{X}(x)\right)\left(1-F_{W}(t)\right), \\
f_{X, W}(x, t)=\lambda e^{-\lambda t} f(x)+\theta\left(2 \lambda e^{-2 \lambda t}-\lambda e^{-\lambda t}\right) h(x),
\end{gathered}
$$

where $h(x)=\left(1-2 F_{X}(x)\right) f(x)$, with Laplace transform (LT) $h_{x}^{*}$.

In the rest of this paper, we assume that $\left\{\left(X_{i}, W_{i}\right), i \in\right.$ $\left.N^{+}\right\}$form a sequence of i.i.d. random vectors distributed like $(X, W)$, which have joint c.d.f. and p.d.f. given by (6) and (7), respectively. In particular, we know from (7) that the conditional p.d.f. of the claim size is given by

$$
f_{X \mid W=t}(x)=f(x)+\theta\left(2 \lambda e^{-\lambda t}-1\right) h(x) .
$$

Also, we assume that $\theta \neq 0$; otherwise our model reduces to the constant dividend barrier in the classical risk model.

The total claim amount process $\{S(t), t \geq 0\}$ is defined as $S(t)=\sum_{i=1}^{N(t)} X_{i}$; let $U_{b}(0)=u$ and

$$
\begin{gathered}
d U_{b}(t)=c d t-d S(t), \quad \text { if } U_{b}(t)<b \\
d U_{b}(t)=-d S(t), \quad \text { if } U_{b}(t)=b
\end{gathered}
$$

be the surplus process in the presence of a constant dividend barrier $b(0<b<\infty)$, where $u \geq 0$ is the initial surplus level and $c(c>0)$ is the level premium. In other words, we assume that the insurer pays the premium rate $c$ as a dividend 
whenever the insurer's surplus remains at the threshold level $b$.

Associated with the risk model, we denote the ruin time by $T$, which is the first passage time of $U_{b}(t)$ below zero level; that is,

$$
T=\inf \left\{t \geq 0, U_{b}(t)<0\right\}
$$

with $T=\infty$ if $U_{b}(t) \geq 0$, for all $t \geq 0$. To guarantee that ruin is not a certain event, we assume that the following net profit condition holds:

$$
E\left[c W_{i}-X_{i}\right]>0, \quad i=1,2, \ldots
$$

At the same time, we introduce the Gerber-Shiu function defined by

$$
\begin{aligned}
& m_{b, \delta}(u) \\
& =E\left[e^{-\delta T} \omega\left(U\left(T^{-}\right),|U(T)|\right) I(T<\infty) \mid U(0)=u\right],
\end{aligned}
$$

where $\delta \geq 0$ is the force of interest, $I(\cdot)$ is the indicator function, and $\omega\left(U\left(T^{-}\right),|U(T)|\right)$ defined on $[0, \infty) \times(0, \infty)$ is a nonnegative function of the surplus before ruin $U\left(T^{-}\right)$and the deficit at ruin $|U(T)|$.

\section{Gerber-Shiu Discounted Penalty Function}

The main purpose of this section is to derive an integrodifferential equation for the expected discounted penalty function $m_{b, \delta}(u)$, This equation will be useful to derive an explicit solution for $m_{b, \delta}(u)$. Throughout this paper, we denote I and $\mathrm{D}$ to be the identity and the differential operators, respectively.

Theorem 1. In the compound Poisson risk model with a dependence structure based on FGM copula defined in (2) and a constant dividend $b$, the expected discounted penalty function $m_{b, \delta}(u)$ satisfies the following integrodifferential equation:

$$
\begin{aligned}
& \left(\frac{2 \lambda+\delta}{c} \mathbf{I}-\mathbf{D}\right)\left(\frac{\lambda+\delta}{c} \mathbf{I}-\mathbf{D}\right) m_{b, \delta}(u) \\
& =\frac{\lambda}{c}\left(\frac{2 \lambda+\delta}{c} \mathbf{I}-\mathbf{D}\right) \sigma_{1}(u)+\frac{\theta \lambda}{c}\left(\frac{\delta}{c} \mathbf{I}-\mathbf{D}\right) \sigma_{2}(u)
\end{aligned}
$$

for $0 \leq u \leq b<\infty$ with boundary conditions:

$$
\begin{gathered}
m_{b, \delta}^{\prime}(b)=0, \\
m_{b, \delta}^{\prime \prime}(b)=-\frac{\lambda}{c} \sigma_{1}^{\prime}(b)-\frac{\theta \lambda}{c} \sigma_{2}^{\prime}(b),
\end{gathered}
$$

where

$$
\begin{gathered}
\sigma_{1}(u)=\int_{0}^{u} m_{b, \delta}(u-x) f(x) d x+\omega_{1}(u), \\
\sigma_{2}(u)=\int_{0}^{u} m_{b, \delta}(u-x) h(x) d x+\omega_{2}(u), \\
\omega_{1}(u)=\int_{u}^{\infty} \omega(u, x-u) f(x) d x, \\
\omega_{2}(u)=\int_{u}^{\infty} \omega(u, x-u) h(x) d x .
\end{gathered}
$$

Proof. By conditioning on the time and the amount of the first claim, we have

$$
\begin{aligned}
m_{b, \delta}(u)= & \int_{0}^{(b-u) / c} \int_{0}^{u+c t} e^{-\delta t} m_{b, \delta}(u+c t-x) f_{X, W}(x, t) d x d t \\
& +\int_{0}^{(b-u) / c} \int_{u+c t}^{\infty} e^{-\delta t} \times \omega(u+c t, x-u-c t) \\
& \times f_{X, W}(x, t) d x d t \\
& +\int_{(b-u) / c}^{\infty} \int_{0}^{b} e^{-\delta t} m_{b, \delta}(b-x) f_{X, W}(x, t) d x d t \\
& +\int_{(b-u) / c}^{\infty} \int_{b}^{\infty} e^{-\delta t} \omega(b, x-b) f_{X, W}(x, t) d x d t
\end{aligned}
$$

Given from (7), (20) becomes

$$
\begin{aligned}
m_{b, \delta}(u)= & \lambda \int_{0}^{(b-u) / c} e^{-(\lambda+\delta) t} \sigma_{1}(u+c t) d t \\
& +2 \theta \lambda \int_{0}^{(b-u) / c} e^{-(2 \lambda+\delta) t} \sigma_{2}(u+c t) d t \\
& -\theta \lambda \times \int_{0}^{(b-u) / c} e^{-(\lambda+\delta) t} \sigma_{2}(u+c t) d t \\
& +\lambda \int_{(b-u) / c}^{\infty} e^{-(\lambda+\delta) t} \sigma_{1}(b) d t \\
& +2 \theta \lambda \int_{(b-u) / c}^{\infty} e^{-(2 \lambda+\delta) t} \sigma_{2}(b) d t \\
& -\theta \lambda \int_{(b-u) / c}^{\infty} e^{-(\lambda+\delta) t} \sigma_{2}(b) d t
\end{aligned}
$$

where the functions $\sigma_{1}(u)$ and $\sigma_{2}(u)$ are given in (16) and (17), respectively.

Simple modifications of (21) lead to

$$
\begin{aligned}
m_{b, \delta}(u)= & \frac{\lambda}{c} \int_{u}^{b} e^{-(\lambda+\delta)((t-u) / c)} \sigma_{1}(t) d t \\
& +\frac{2 \theta \lambda}{c} \int_{u}^{b} e^{-(2 \lambda+\delta)((t-u) / c)} \sigma_{2}(t) d t \\
& -\frac{\theta \lambda}{c} \int_{u}^{b} e^{-(\lambda+\delta)((t-u) / c)} \times \sigma_{2}(t) d t \\
& +\frac{\lambda}{c} \int_{b}^{\infty} e^{-(\lambda+\delta)((t-u) / c)} \sigma_{1}(b) d t \\
& +\frac{2 \theta \lambda}{c} \int_{b}^{\infty} e^{-(2 \lambda+\delta)((t-u) / c)} \sigma_{2}(b) d t \\
& -\frac{\theta \lambda}{c} \int_{b}^{\infty} e^{-(\lambda+\delta)((t-u) / c)} \sigma_{2}(b) d t .
\end{aligned}
$$


We can rewrite $(22)$ as

$$
\begin{aligned}
m_{b, \delta}(u)= & \frac{\lambda}{c} \int_{u}^{\infty} e^{-(\lambda+\delta)((t-u) / c)} \sigma_{1}(t \wedge b) d t \\
& +\frac{2 \theta \lambda}{c} \int_{u}^{\infty} e^{-(2 \lambda+\delta)((t-u) / c)} \sigma_{2}(t \wedge b) d t \\
& -\frac{\theta \lambda}{c} \times \int_{u}^{\infty} e^{-(\lambda+\delta)((t-u) / c)} \sigma_{2}(t \wedge b) d t,
\end{aligned}
$$

where $t \wedge b=\min (t, b)$.

Now differentiating (23) with respect to $u$, routine calculations lead to

$$
\begin{aligned}
m_{b, \delta}^{\prime}(u)= & \frac{\lambda+\delta}{c} \frac{\lambda}{c} \int_{u}^{\infty} e^{-(\lambda+\delta)((t-u) / c)} \sigma_{1}(t \wedge b) d t-\frac{\lambda}{c} \sigma_{1}(u) \\
& +\frac{2 \lambda+\delta}{c} \frac{2 \theta \lambda}{c} \int_{u}^{\infty} e^{-(2 \lambda+\delta)((t-u) / c)} \sigma_{2}(t \wedge b) d t \\
& -\frac{\theta \lambda}{c} \sigma_{2}(u)-\frac{\lambda+\delta}{c} \frac{\theta \lambda}{c} \int_{u}^{\infty} e^{-(\lambda+\delta)((t-u) / c)} \\
& \times \sigma_{2}(t \wedge b) d t
\end{aligned}
$$

Substituting (23) into (24), we obtain

$$
\begin{aligned}
& m_{b, \delta}^{\prime}(u) \\
& =\frac{\lambda+\delta}{c}\left[m_{b, \delta}(u)-\frac{2 \theta \lambda}{c} \int_{u}^{\infty} e^{-(2 \lambda+\delta)((t-u) / c)} \sigma_{2}(t \wedge b) d t\right. \\
& \left.\quad+\frac{\theta \lambda}{c} \int_{u}^{\infty} e^{-(\lambda+\delta)((t-u) / c)} \sigma_{2}(t \wedge b) d t\right] \\
& +\frac{2 \lambda+\delta}{c} \frac{2 \theta \lambda}{c} \int_{u}^{\infty} e^{-(2 \lambda+\delta)((t-u) / c)} \sigma_{2}(t \wedge b) d t \\
& -\frac{\lambda+\delta}{c} \frac{\theta \lambda}{c} \int_{u}^{\infty} e^{-(\lambda+\delta)((t-u) / c)} \sigma_{2}(t \wedge b) d t \\
& -\frac{\lambda}{c} \sigma_{1}(u)-\frac{\theta \lambda}{c} \sigma_{2}(u) .
\end{aligned}
$$

That is,

$$
\begin{aligned}
& m_{b, \delta}^{\prime}(u) \\
& =\frac{\lambda+\delta}{c} m_{b, \delta}(u)+\frac{\lambda}{c} \frac{2 \theta \lambda}{c} \int_{u}^{\infty} e^{-(2 \lambda+\delta)((t-u) / c)} \sigma_{2}(t \wedge b) d t \\
& -\frac{\lambda}{c} \sigma_{1}(u)-\frac{\theta \lambda}{c} \sigma_{2}(u) .
\end{aligned}
$$

Differentiating (26) with respect to $\mathrm{u}$, we find

$$
\begin{aligned}
m_{b, \delta}^{\prime \prime}(u)= & \frac{\lambda+\delta}{c} m_{b, \delta}^{\prime}(u)+\frac{\delta+2 \lambda}{c} \frac{\lambda}{c} \frac{2 \theta \lambda}{c} \\
& \times \int_{u}^{\infty} e^{-(2 \lambda+\delta)((t-u) / c)} \sigma_{2}(t \wedge b) d t \\
& -\frac{\lambda}{c} \frac{2 \theta \lambda}{c} \sigma_{2}(u)-\frac{\lambda}{c} \sigma_{1}^{\prime}(u)-\frac{\theta \lambda}{c} \sigma_{2}^{\prime}(u)
\end{aligned}
$$

which can be reexpressed as

$$
\begin{aligned}
m_{b, \delta}^{\prime \prime}(u) & \\
= & \frac{\lambda+\delta}{c} m_{b, \delta}^{\prime}(u)+\frac{\delta+2 \lambda}{c} \\
& \times\left[m_{b, \delta}^{\prime}(u)-\frac{\lambda+\delta}{c} m_{b, \delta}(u)+\frac{\lambda}{c} \sigma_{1}(u)+\frac{\theta \lambda}{c} \sigma_{2}(u)\right] \\
& -\frac{\lambda}{c} \frac{2 \theta \lambda}{c} \sigma_{2}(u)-\frac{\lambda}{c} \sigma_{1}^{\prime}(u)-\frac{\theta \lambda}{c} \sigma_{2}^{\prime}(u) .
\end{aligned}
$$

That is,

$$
\begin{aligned}
m_{b, \delta}^{\prime \prime}(u)= & \frac{3 \lambda+2 \delta}{c} m_{b, \delta}^{\prime}(u)-\frac{\lambda+\delta}{c} \frac{\delta+2 \lambda}{c} m_{b, \delta}(u) \\
& +\frac{\lambda}{c} \frac{\delta+2 \lambda}{c} \sigma_{1}(u)-\frac{\lambda}{c} \sigma_{1}^{\prime}(u)+\frac{\theta \lambda}{c} \frac{\delta}{c} \sigma_{2}(u) \\
& -\frac{\theta \lambda}{c} \sigma_{2}^{\prime}(u)
\end{aligned}
$$

Using the identical and differential operators, we obtain (13).

Regarding the boundary conditions, (14) is derived from (24) at $u=b$. While (15) can be proven via (27) at $u=b$ and (14).

Note that (13) in itself does not depend on the barrier level $b$, therefore, one concludes that $m_{\infty, \delta}(u)$, the GerberShiu discounted penalty function in the absence of a barrier, satisfies the second order nonhomogeneous integrodifferential equation:

$$
\begin{aligned}
& \left(\frac{2 \lambda+\delta}{c} \mathbf{I}-\mathbf{D}\right)\left(\frac{\lambda+\delta}{c} \mathbf{I}-\mathbf{D}\right) m_{\infty, \delta}(u) \\
& =\frac{\lambda}{c}\left(\frac{2 \lambda+\delta}{c} \mathbf{I}-\mathbf{D}\right) \sigma_{3}(u)+\frac{\theta \lambda}{c}\left(\frac{\delta}{c} \mathbf{I}-\mathbf{D}\right) \sigma_{4}(u) ;
\end{aligned}
$$

where

$$
\begin{aligned}
& \sigma_{3}(u)=\int_{0}^{u} m_{\infty, \delta}(u-x) f(x) d x+\omega_{1}(u), \\
& \sigma_{4}(u)=\int_{0}^{u} m_{\infty, \delta}(u-x) h(x) d x+\omega_{2}(u) .
\end{aligned}
$$

As shown in Cossette et al. [7], it is a solution to a defective renewal equation.

\section{A Representation of \\ the Discounted Penalty Function}

In the present section, we derive the defective renewal equation for $m_{b, \delta}(u)$. For that purpose, we use the DicksonHipp operator $T_{s}$ for an integrable real-valued function $f$ (introduced by Dickson and Hipp (2001)) defined by

$$
T_{s} f(x)=\int_{x}^{\infty} e^{-s(y-x)} f(y) d y, \quad s \in C .
$$


The operator $T_{s}$ is commutative; that is, $T_{r} T_{s}=T_{s} T_{r}$; moreover,

$$
T_{s} T_{r} f(x)=T_{r} T_{s} f(x)=\frac{T_{s} f(x)-T_{r} f(x)}{r-s}, \quad s \neq r .
$$

From Theorem 1, one concludes that $m_{b, \delta}(u)$ satisfies a nonhomogeneous equation of order 2. From the theory on differential equations, the solution to the second order nonhomogeneous equation (13) for $m_{b, \delta}(u)$ (with boundary conditions (14) and (15)) can be expressed as a particular solution $m_{\infty, \delta}(u)$ and a given combination of two linearly independent solutions to the associated homogeneous integrodifferential equation:

$$
\begin{aligned}
&\left(\frac{2 \lambda}{c}+\delta\right.\mathbf{I}-\mathbf{D})\left(\frac{\lambda+\delta}{c} \mathbf{I}-\mathbf{D}\right) y(u) \\
&= \frac{\lambda}{c}\left(\frac{2 \lambda+\delta}{c} \mathbf{I}-\mathbf{D}\right) \int_{0}^{u} y(u-x) f(x) d x+\frac{\theta \lambda}{c} \\
& \quad \times\left(\frac{\delta}{c} \mathbf{I}-\mathbf{D}\right) \int_{0}^{u} y(u-x) h(x) d x .
\end{aligned}
$$

By letting $y^{*}(s)=\int_{0}^{\infty} e^{-s x} y(x) d x$, let us take Laplace transform on the both sides of the homogeneous equation (34). We can obtain

$$
\begin{aligned}
& y^{*}(s) \\
& =\left(\left(s-\frac{3 \lambda+2 \delta}{c}\right) y(0)+y^{\prime}(0)\right) \\
& \quad \times\left(\left(\frac{2 \lambda+\delta}{c}-s\right)\left(\frac{\lambda+\delta}{c}-s\right)-\frac{\lambda}{c}\right. \\
& \left.\quad \times\left[\left(\frac{2 \lambda+\delta}{c}-s\right) f^{*}(s)+\theta\left(\frac{\delta}{c}-s\right) h^{*}(s)\right]\right)^{-1} .
\end{aligned}
$$

From (35), it is clear that the solution to (34) can be written as a combination of the two linearly independent solutions $\left\{y_{1, \delta}(u), u \geq 0\right\}$ and $\left\{y_{2, \delta}(u), u \geq 0\right\}$, where

$$
\begin{aligned}
& y_{1, \delta}^{*}(s) \\
& =\left(s-\frac{3 \lambda+2 \delta}{c}\right) \\
& \quad \times\left(\left(\frac{2 \lambda+\delta}{c}-s\right)\left(\frac{\lambda+\delta}{c}-s\right)-\frac{\lambda}{c}\right. \\
& \left.\quad \times\left[\left(\frac{2 \lambda+\delta}{c}-s\right) f^{*}(s)+\theta\left(\frac{\delta}{c}-s\right) h^{*}(s)\right]\right)^{-1},
\end{aligned}
$$

with $y_{1, \delta}(0)=1$ and $y_{1, \delta}^{\prime}(0)=0$, and

$$
\begin{aligned}
y_{2, \delta}^{*}(s)= & \\
& \times\left(\left(\frac{2 \lambda+\delta}{c}-s\right)\left(\frac{\lambda+\delta}{c}-s\right)-\frac{\lambda}{c}\right. \\
& \left.\times\left[\left(\frac{2 \lambda+\delta}{c}-s\right) f^{*}(s)+\theta\left(\frac{\delta}{c}-s\right) h^{*}(s)\right]\right)^{-1},
\end{aligned}
$$

with $y_{2, \delta}(0)=0$ and $y_{2, \delta}^{\prime}(0)=1$.

Theorem 2. For the Gerber-Shiu discounted penalty function satisfying (13), a closed-form expression for $m_{b, \delta}(u)$ is given by

$$
m_{b, \delta}(u)=m_{\infty, \delta}(u)+\xi_{1} y_{1, \delta}(u)+\xi_{2} y_{2, \delta}(u), \quad 0 \leq u \leq b,
$$

where the constants $\xi_{1}, \xi_{2}$ are the solutions to the following system of linear equations:

$$
\begin{gathered}
\xi_{1} y_{1, \delta}^{\prime}(b)+\xi_{2} y_{2, \delta}^{\prime}(b)=-m_{\infty, \delta}^{\prime}(b), \\
\xi_{1}\left(y_{1, \delta}^{\prime \prime}(b)+\left.\frac{\lambda}{c} \mathbf{D} \int_{0}^{u} y_{1, \delta}(u-x) f(x) d x\right|_{u=b}\right) \\
+\left.\frac{\theta \lambda}{c} \mathbf{D} \int_{0}^{u} y_{1, \delta}(u-x) h(x) d x\right|_{u=b} \\
+\xi_{2}\left(y_{2, \delta}^{\prime \prime}(b)+\left.\frac{\lambda}{c} \mathbf{D} \int_{0}^{u} y_{2, \delta}(u-x) f(x) d x\right|_{u=b}\right) \\
+\left.\frac{\theta \lambda}{c} \mathbf{D} \int_{0}^{u} y_{2, \delta}(u-x) h(x) d x\right|_{u=b} \\
=-\left[m_{\infty, \delta}^{\prime \prime}(b)+\left.\frac{\lambda}{c} \mathbf{D} \int_{0}^{u} m_{\infty, \delta}(u-x) f(x) d x\right|_{u=b}\right. \\
+\left.\frac{\theta \lambda}{c} \mathbf{D} \int_{0}^{u} m_{\infty, \delta}(u-x) h(x) d x\right|_{u=b} \\
\left.+\frac{\lambda}{c} \omega_{1}^{\prime}(b)+\frac{\theta \lambda}{c} \omega_{2}^{\prime}(b)\right] .
\end{gathered}
$$

Proof. It is immediate that $m_{b, \delta}(u)$ is of the form

$$
m_{b, \delta}(u)=m_{\infty, \delta}(u)+\xi_{1} y_{1, \delta}(u)+\xi_{2} y_{2, \delta}(u)
$$

Thus, by (14) and (15), differentiating (41) with respect to $u$ at $u=b$, we obtain

$$
\begin{gathered}
m_{\infty, \delta}^{\prime}(b)+\xi_{1} y_{1, \delta}^{\prime}(b)+\xi_{2} y_{2, \delta}^{\prime}(b)=0 \\
m_{\infty, \delta}^{\prime \prime}(b)+\xi_{1} y_{1, \delta}^{\prime \prime}(b)+\xi_{2} y_{2, \delta}^{\prime \prime}(b)=-\frac{\lambda}{c} \sigma_{1}^{\prime}(b)-\frac{\theta \lambda}{c} \sigma_{2}^{\prime}(b) .
\end{gathered}
$$

Equation (42) is equivalent to (39). 
Using the structural form (38) for $m_{b, \delta}(u)$, differentiation with respect to $u$ of (16) and (17) yields

$$
\begin{aligned}
\mathbf{D}\left(\sigma_{1}(u)\right)= & \xi_{1} \mathbf{D} \int_{0}^{u} y_{1, \delta}(u-x) f(x) d x \\
& +\xi_{2} \mathbf{D} \int_{0}^{u} y_{2, \delta}(u-x) f(x) d x \\
& +\mathbf{D} \int_{0}^{u} m_{\infty, \delta}(u-x) f(x) d x+\mathbf{D} \omega_{1}(u) \\
\mathbf{D}\left(\sigma_{2}(u)\right)= & \xi_{1} \mathbf{D} \int_{0}^{u} y_{1, \delta}(u-x) h(x) d x \\
& +\xi_{2} \mathbf{D} \int_{0}^{u} y_{2, \delta}(u-x) h(x) d x \\
& +\mathbf{D} \int_{0}^{u} m_{\infty, \delta}(u-x) h(x) d x+\mathbf{D} \omega_{2}(u) .
\end{aligned}
$$

Substituting (44) into the right-hand side of (43) at $u=b$ leads to (40).

From Propositions 4.1 and 4.2 of Cossette et al. [7], we know that the denominator on the right-hand side of (36) and (37) has only two positive, real, and distinct roots, say, $s_{1}$ and $s_{2}$.

Using (47) of Cossette et al. [7], (36) and (37) can be expressed as

$$
\begin{gathered}
y_{1, \delta}^{*}(s) \\
=\left(\left(\left(s_{1}-\frac{3 \lambda+2 \delta}{c}\right) \frac{s-s_{1}}{s_{2}-s_{1}}+\left(s_{2}-\frac{3 \lambda+2 \delta}{c}\right) \frac{s-s_{2}}{s_{1}-s_{2}}\right)\right. \\
\left.\quad \times\left(\left(s-s_{1}\right)\left(s-s_{2}\right)\right)^{-1}\right)\left(1-T_{s} T_{s_{2}} T_{s_{1}} h_{2, \delta}(0)\right)^{-1} \\
y_{2, \delta}^{*}(s)=\frac{1 /\left(s-s_{1}\right)\left(s-s_{2}\right)}{1-T_{s} T_{s_{2}} T_{s_{1}} h_{2, \delta}(0)}
\end{gathered}
$$

where

$$
h_{2, \delta}^{*}(s)=\frac{\lambda}{c}\left[\left(\frac{2 \lambda+\delta}{c}-s\right) f^{*}(s)+\theta\left(\frac{\delta}{c}-s\right) h^{*}(s)\right] .
$$

Therefore, the Laplace transform (45) and (46) can now be used to find an expression for the two linearly independent solutions $\left\{y_{1, \delta}(u), u \geq 0\right\}$ and $\left\{y_{2, \delta}(u), u \geq 0\right\}$, respectively. From Proposition 7.2 of Cossette et al. [7], (45) and (46) lead to

$$
\begin{aligned}
y_{1, \delta}(u)= & k_{\delta} \int_{0}^{u} y_{1, \delta}(u-y) g_{\delta}(y) d y+\frac{s_{1}-(3 \lambda+2 \delta) / c}{s_{2}-s_{1}} e^{s_{2} u} \\
& +\frac{s_{2}-(3 \lambda+2 \delta) / c}{s_{1}-s_{2}} e^{s_{1} u} \\
y_{2, \delta}(u) & =k_{\delta} \int_{0}^{u} y_{2, \delta}(u-y) g_{\delta}(y) d y+\frac{e^{s_{2} u}-e^{s_{1} u}}{s_{2}-s_{1}}
\end{aligned}
$$

where

$$
\begin{gathered}
k_{\delta}=\frac{\lambda}{c}\left[\left(\frac{\delta+2 \lambda}{c}-s_{2}\right) T_{0} T_{s_{2}} T_{s_{1}} f(0)+\theta\left(\frac{\delta}{c}-s_{2}\right)\right. \\
\left.\times T_{0} T_{s_{2}} T_{s_{1}} h(0)+T_{0} T_{s_{1}} f(0)+\theta T_{0} T_{s_{1}} h(0)\right], \\
g_{\delta}(y)=\frac{T_{s_{2}} T_{s_{1}} h_{2, \delta}(u)}{k_{\delta}} .
\end{gathered}
$$

The defective renewal equations (48) and (49) may be solved to give an explicit for $y_{1, \delta}(u)$ and $y_{2, \delta}(u)$. By a similar way to the one used in Landriault [10], we choose

$$
L_{\delta}(u)=1-\sum_{n=1}^{\infty}\left(1-k_{\delta}\right)\left(k_{\delta}\right)^{n} \bar{G}_{\delta}^{* n}(y)
$$

where $\bar{G}_{\delta}^{* n}(y)$ is the survival distribution of the $n$-fold convolution of the p.d.f. $g_{\delta}(y)$.

Theorem 3. Let $\lambda_{i, \delta}(u)=e^{s_{i} u}-s_{i} \int_{0}^{u} e^{s_{i} y} L_{\delta}(u-y) d y$ for $i=1,2$. The solutions to (48) and (49) mat be expressed respectively as follows:

$$
\begin{aligned}
& y_{1, \delta}(u)=\left(s_{1}\left(\lambda_{1, \delta}(u)-L_{\delta}(u)\right)-s_{2}\left(\lambda_{2, \delta}(u)-L_{\delta}(u)\right)\right. \\
&\left.-\frac{3 \lambda+2 \delta}{c}\left(\lambda_{1, \delta}(u)-\lambda_{2, \delta}(u)\right)\right) \\
& \times\left(\left(1-k_{\delta}\right)\left(s_{2}-s_{1}\right)\right)^{-1} \\
& y_{2, \delta}(u)=\frac{\lambda_{1, \delta}(u)-\lambda_{2, \delta}(u)}{\left(1-k_{\delta}\right)\left(s_{2}-s_{1}\right)} .
\end{aligned}
$$

Proof. Applying Theorem 9.2 of Willmot and Lin [22] to the defective renewal equation (48) and (49), respectively, we can obtain (52) and (53) immediately.

All above derivations can derive the closed-form expression for $m_{b, \delta}(u)$ by (38).

\section{Conflict of Interests}

The authors declare no conflict of interests regarding the publication for the paper.

\section{Acknowledgments}

This research is fully supported by a Grant from Natural Science foundation of Hunan (13JJ4083), by Humanities and Social Sciences project of the Ministry Education in China (10YJC630144), and by Hunan Social Science Fund Program (12YBB093), and it is also supported by Scientific Research Fund of Hunan Provincial Education Department (13C318).

\section{References}

[1] H. U. Gerber and E. S. W. Shiu, "On the time value of ruin," North American Actuarial Journal, vol. 2, no. 1, pp. 48-78, 1998. 
[2] M. Boudreault, "Modeling and pricing earthquake risk," Scor Canada Actuarial Prize, 2003.

[3] A. K. Nikoloulopoulos and D. Karlis, "Fitting copulas to bivariate earthquake data: the seismic gap hypothesis revisited," Environmetrics, vol. 19, no. 3, pp. 251-269, 2008.

[4] H. Albrecher and J. L. Teugels, "Exponential behavior in the presence of dependence in risk theory," Journal of Applied Probability, vol. 43, no. 1, pp. 257-273, 2006.

[5] M. Boudreault, H. Cossette, D. Landriault, and E. Marceau, "On a risk model with dependence between interclaim arrivals and claim sizes," Scandinavian Actuarial Journal, vol. 5, pp. 301-323, 2006.

[6] E. Marceau, "On the discrete-time compound renewal risk model with dependence," Insurance: Mathematics \& Economics, vol. 44, no. 2, pp. 245-259, 2009.

[7] H. Cossette, E. Marceau, and F. Marri, "Analysis of ruin measures for the classical compound Poisson risk model with dependence," Scandinavian Actuarial Journal, vol. 2010, no. 3, pp. 221-245, 2010.

[8] Z. Zhang and H. Yang, "Gerber-Shiu analysis in a perturbed risk model with dependence between claim sizes and interclaim times," Journal of Computational and Applied Mathematics, vol. 235, no. 5, pp. 1189-1204, 2011.

[9] B. de Finetti, "Su un'impostazione alternativa dell teoria colletiva del rischio," Transactions of the XV International Congress of Actuaries, vol. 2, pp. 433-443, 1957.

[10] D. Landriault, "Constant dividend barrier in a risk model with interclaim-dependent claim sizes," Insurance: Mathematics \& Economics, vol. 42, no. 1, pp. 31-38, 2008.

[11] H. Albrecher, J. Hartinger, and R. F. Tichy, "On the distribution of dividend payments and the discounted penalty function in a risk model with linear dividend barrier," Scandinavian Actuarial Journal, vol. 2005, no. 2, pp. 103-126, 2005.

[12] K. C. Yuen, G. Wang, and W. K. Li, "The Gerber-Shiu expected discounted penalty function for risk processes with interest and a constant dividend barrier," Insurance: Mathematics \& Economics, vol. 40, no. 1, pp. 104-112, 2007.

[13] D. C. M. Dickson and H. R. Waters, "Some optimal dividends problems," Astin Bulletin, vol. 34, no. 1, pp. 49-74, 2004.

[14] X. S. Lin, G. E. Willmot, and S. Drekic, "The classical risk model with a constant dividend barrier: analysis of the GerberShiu discounted penalty function," Insurance: Mathematics and Economics, vol. 33, no. 3, pp. 551-566, 2003.

[15] C. Segerdahl, "On some distributions in time connected with the collective theory of risk," Scandinavian Actuarial Journal, pp. 167-192, 1970.

[16] Y. Chi and X. S. Lin, "On the threshold dividend strategy for a generalized jump-diffusion risk model," Insurance: Mathematics and Economics, vol. 48, no. 3, pp. 326-337, 2011.

[17] D. Liu and Z. Liu, "The perturbed compound Poisson risk model with linear dividend barrier," Journal of Computational and Applied Mathematics, vol. 235, no. 8, pp. 2357-2363, 2011.

[18] M. Bratiichuk, "On the Gerber-Shiu function for a risk model with multi-layer dividend strategy," Statistics \& Probability Letters, vol. 82, no. 3, pp. 496-504, 2012.

[19] S. Chadjiconstantinidis and A. D. Papaioannou, "On a perturbed by diffusion compound Poisson risk model with delayed claims and multi-layer dividend strategy," Journal of Computational and Applied Mathematics, vol. 253, pp. 26-50, 2013.

[20] W. Wang, “The perturbed sparre andersen model with interest and a threshold dividend strategy," Methodology and Computing in Applied Probability, 2013.
[21] R. B. Nelsen, An Introduction to Copulas, Springer, New York, NY, USA, 2nd edition, 2006.

[22] G. E. Willmot and S. Lin, Lundberg Approximations for Compound Distributions with Insurance Applications, Lecture Notes in Statistics, Springe, New York, NY, USA, 2001. 


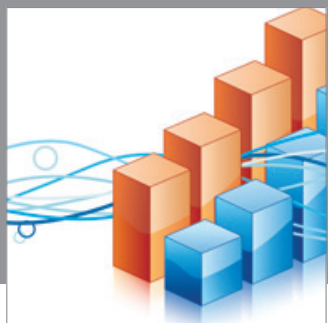

Advances in

Operations Research

mansans

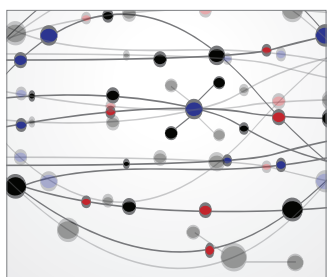

The Scientific World Journal
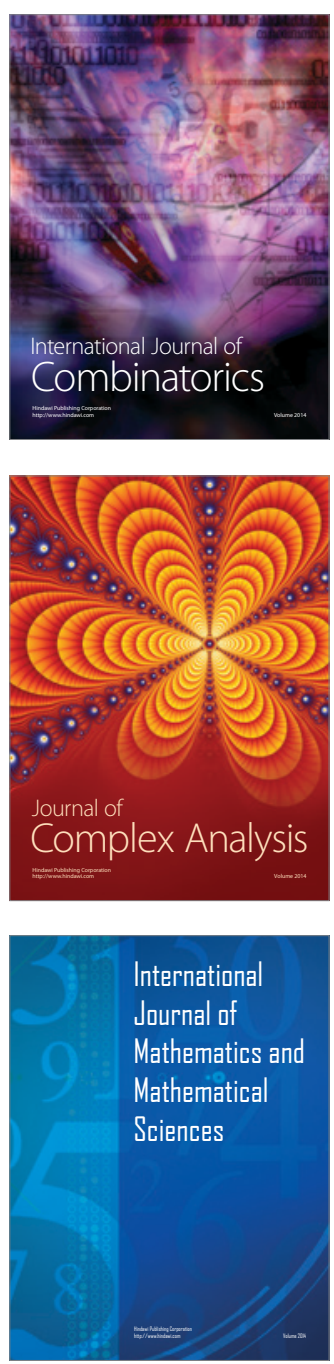
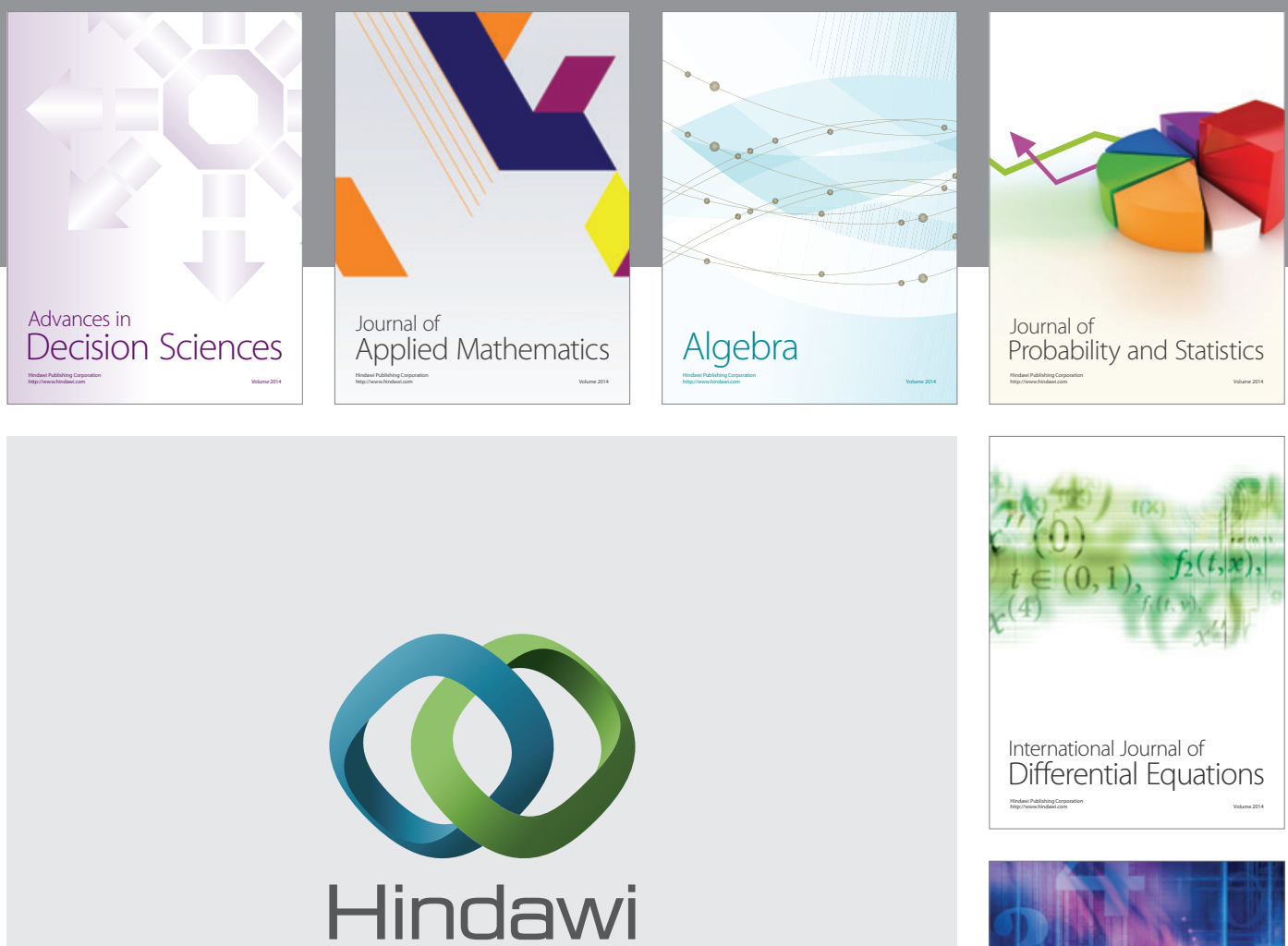

Submit your manuscripts at http://www.hindawi.com
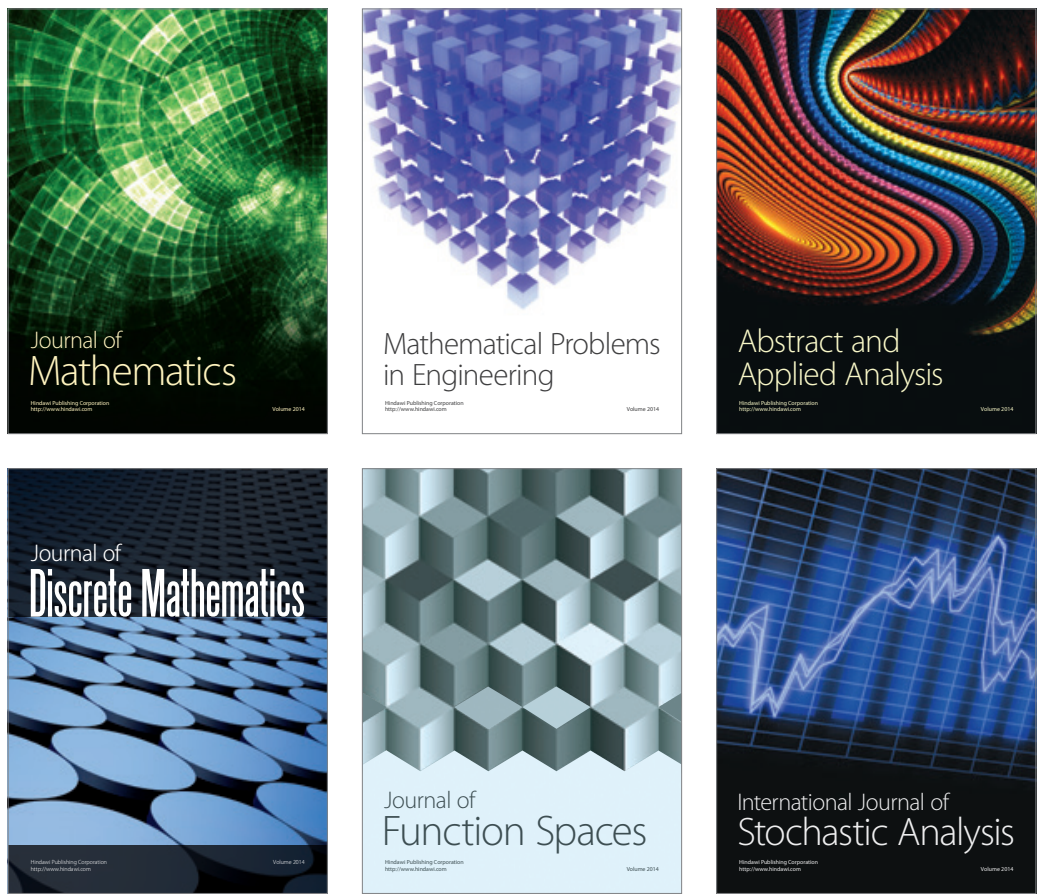

Journal of

Function Spaces

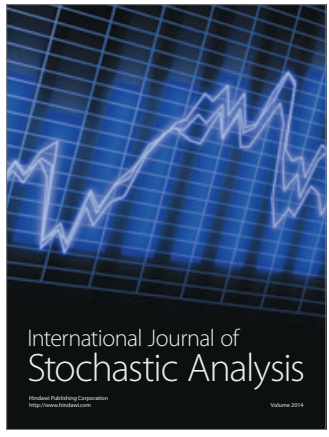

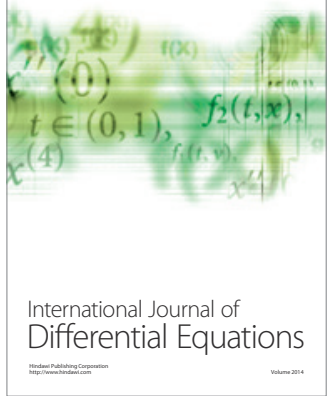
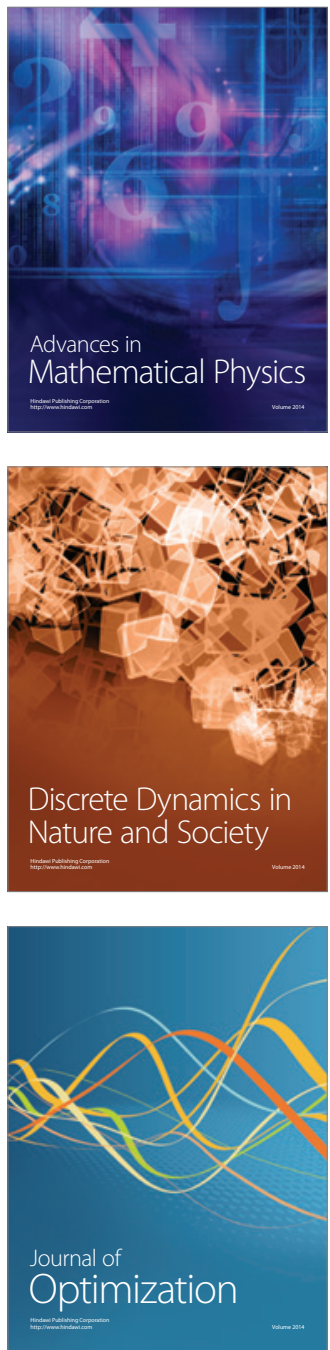